\title{
Frequency Reserve Within Unit Commitment Considering Spinning Reserve Uncertainty
}

\author{
M.Rouholamini, M.Rashidinejad", A.Abdollahi, H.Ghasemnejad \\ Department of Electrical Engineering, Bahonar University of Kerman, Kerman, Iran
}

\begin{abstract}
Various technical limitations of generating units and ancillary services requirements have more complicated the power generation control and operation. This paper introduces a novel methodology for day-ahead unit commitment, which is a crucial challenge in restructured power systems, considering primary frequency control reserve. In addition, also spinning reserve uncertainty as a practical constraint has been taken into account.

In this paper, we formulate and solve simultaneous scheduling of energy and primary reserve as a mixed integer non linear programming problem that simultaneously accounts aforementioned constraints. In proposed formulation, system frequency is allowed to fall from nominal value to a critical specified limit.

Finally, the proposed approach is implemented to the scheduling of a 17-unit isolated power system over 24-hours. Case studies and numerical results present significant outcomes and verify the robustness of the proposed method, while it creates a schedule consistent with the primary frequency control and spinning reserve uncertainty.
\end{abstract}

Keywords Frequency Deviation, GAMS, Load Ramp Rate, MINLP, Primary Reserve, Simultaneous Scheduling, Spinning Reserve Probability

\section{Introduction}

A power system is always exposed to sudden variation, unwanted changes in system demand or losing generating units that cause a frequency deviation in system frequency. In particularly, a change in system load or loss a generating unit will results in a steady-state frequency deviation, depending on the governor droop characteristic. So the frequency regulating will be arisen. Frequency regulating is one of most important challenges for Independent System Operator (ISO, hereinafter) or market operator in deregulate d electricity industry. In restructured power systems, frequency regulating can be considered as an ancillary service (AS, hereinafter) that will be supplied employing the eligible resources provided by market participants. Usually, preparation reserve capability is called as "frequency control reserve", which is classified as primary, secondary and tertiary reserve. This categorization depends on the response time of frequency control reserves and how they are deployed[1],[2]. Primary frequency reserve is madeavailabl e by a set of individual participating generators. Actually the primary reserve is obtained mainly from the response of governor droop characteristic to system frequency deviation from nominal. This is the fastest of the three aforementioned

* Corresponding author:

mehdi.amini82@gmail.com(M.Rashidinejad)

Published online at http://journal.sapub.org/ijee

Copyright (C) 2012 Scientific \& Academic Publishing. All Rights Reserved reserve control strategies with a response time of the order of seconds[3]. The secondary reserve, called as Automatic Generation Control (AGC, hereinafter) and more specificall y Load Frequency Control (LFC) is with a response time of the order of minutes. AGC is applied to regulate the area control-error. Area control -error is defined as tie-lines flow violation versus frequency deviation in a multi-area power network[4]. Secondary reserve is with a response time reported as one minute. The tertiary reserve, with a response time of the order of minutes, can be used for congestion management, improvement of lost reserves and compensati on of the incomplete tasks that has not completely been done by AGC. In this paper, we just emphasized the primary reserve without considering the secondary and tertiary reserves.

Previous researches indicate that scheduling of energy and AS are developed using simultaneously or sequentially methods in different countries[3],[4]. Form market point of view, energy and AS are transacted simultaneously while technically ancillary services will be produced after prepara tion of energy in sequential scheduling. Using sequential scheduling method, it is possible that "price reversal" be occurred, where this event has been experienced in California and Newengland[5]. Simultaneous scheduling is more complex in comparison with sequential scheduling but it may converge to a global optimal solution. In addition, it is possible, the final solution of sequential method may be not applicable[5].

Reference[6] is one of most important researches about scheduling of energy and primary reserves which has 
employed an iterative economic dispatch and in it; the generation output and reserve of each unit are modified successively. $\operatorname{In}[7]$, operational and stability constraints have been considered in scheduling problem using decision tree solution method. However, in both of the above mentioned approaches, generation is scheduled a priori and then the reserve is scheduled.

Since the type of simultaneous scheduling of energy and frequency reserve is MINLP, in[3], this problem has been solved through converting MINLP into a Mixed Integer Linear Programming (MILP) problem, to be solved by means of commercially available mixed integer software, such as GAMS. This software has been commonly applied to solve many crucial combinatorial optimization problems but the linearization of the quadratic objective function may cause a deviation in final solution. One previous important contribution is the work reported in[8] by Galiana and colleagues. They indicated that simultaneous scheduling of energy and frequency control reserves increases social welfare.

From the aforementioned researches it seems clear that the scheduling of rapid primary frequency regulation reserve within the unit commitment problem has received relatively little attention formulating as simultaneous method.

This paper focuses on simultaneous scheduling of energy and primary reserve and supposes the system is isolated. In the other words the AGC not installed on the system. Here, the contingency has been assumed as the case of lose of one generating unit. This criterion is suggested by North American Electric Reliability Council. In continue, the simultaneous scheduling of energy and primary frequency control reserve is presented and the optimization problem will be formulated as a MINLP problem to be solved using GAMS software. Finally, the effectiveness of the proposed approach is examined on a 17-unit case study in details. A multiperiod unit commitment problem that simultaneously schedules generated power and primary reserves is tested to validate the proposed formulation. This formulation is subject to accounting for spinning reserve uncertainty, start up cost, a security criterion defined by one of prespecified generation outage contingencies and by the explicit requirement that the system frequency must not fall below a specified lower bound under any of the contingencies.

\section{Primary Frequency Control Reserve}

Frequency deviation that stimulates the governor response is mainly caused due to unbalances between generation and demand in power system. Inherent demand randomness is the major reason of these mismatches, which, being generally small and relatively slows, can be corrected by secondary regulation or AGC. Under such conditions, primary regulation is also active, helping keep frequency within bounds, but not in a very conspicuous fashion.

It is obviously, following a large imbalance between demand and generation, for example lose of a generating unit, primary frequency regulation is very essential in limiting frequency deviation and preventing system outage by maintaining a balance between generation and demand. Following lose of a generating unit, system frequency drops from its reference level. Calling this frequency deviation by $\Delta f$, remaining generating units perforce responds through their speed governors by automatically incrementing their outputs according to $-R_{i} \times \Delta f$. $R_{i}$ is the slope of the droop curve and it is generally between 4 and $6 \%$. Incrementing of healthly generating units output will be continued until the system frequency reaches steady-state at a value that causes the sum of the on-line generators output to be equal to the system load. Governor response stabilizes the system frequency within 5 to $10 \mathrm{~s}$. Thus, when referring to system frequency deviation, in this paper, we mean the steady-state value $\Delta f$ reached after the post-contingency transient dies down. We know that with conventional type of governors, when the systems load increases, the system frequency decreases and vice versa. At the same aforementioned important, following losing a generating unit under -frequency relay operation may be occurred. It is clearly, decrease in system frequency versus losing a generating unit must be restricted to a critical specified limit in the steady state, typically around $1 \%$ below the nominal frequency called as $\Delta f_{c r}$ hereinafter. Once the system is in steady state, within minutes, tertiary reserves are deployed to bring the system frequency back to nominal, since secondary reserves and AGC may be insufficient to compensate for the lose of a large generating unit.

The significant time gap between a major contingency and the start of secondary and tertiary corrective actions (in the order of minutes) and preventing under-frequency relay operation means that primary frequency regulation is essentially responsible for initially balancing power and stabilizing the system frequency[3]. It should be noted that loads response to frequency deviation inherently, however it is neglected in following problem formulation.

\section{Problem Formulation}

In this section, problem formulation of simultaneous sche duling of energy and primary reserve considering spinning reserve uncertainty are presented for an isolated power system. The power market in this paper is assumed as pay as bid and the contingency has been modeled as $\mathrm{N}-1$ in formulation.

In continue, formulation of primary reserve and spinning reserve uncertainty are illustrated following general formula tion of unit commitment problem. Finally objective function is expressed to minimize overall cost of both generation and primary reserve provision over the scheduling horizon.

A Unit commitment constraints

The UC problem is subjected to the following constraints [9-13]:

- Power balance constraint 


$$
\sum_{i=1}^{H} u_{i t} \cdot g_{i t}=d_{t}
$$

Here the system loss has been neglected. In condition of considering power losses, total generating output must be equal to demand plus losses during each hour.

- Unit output limits

$$
u_{i t} \cdot g_{i}^{\min } \leq u_{i t} \cdot g_{i t} \leq g_{i}^{\max }
$$

- Unit ramp up constraint

$$
u_{i t} \cdot g_{i t}-u_{i, t-1} \cdot g_{i, t-1} \leq R U R_{i}
$$

- Unit ramp down constraint

$$
u_{i, t-1} \cdot g_{i, t-1}-u_{i t} \cdot g_{i t} \leq R D R_{i}
$$

- Minimum down time limit

$$
M D_{i}^{\text {OFF }} \geq T_{i}^{D}
$$

- Minimum UP time limit

$$
M D_{i}^{O N} \geq T_{i}^{U}
$$

- Initial condition

Initial conditions of generating units include the number of hours that a unit consequently has been on-line or off-line and its generation output at an hour before the scheduling.

- Spinning reserve capacity

Total amount of maximum unit set-point of all committed units minus total demand at each hour is defined as spinning reserve capacity. Spinning reserve must be sufficient enough to maintain the desired reliability of a power system. It is usually a pre-specified limit or equal to the largest unit or a given percentage of the forecasted demand, usually is equal to 10 percent[10],[14],[15].

Spinning reserve constraint can be given by Eq. (7).

$$
\sum_{i=1}^{N} u_{i t} \cdot g_{i}^{\max } \geq d_{t}+S R R_{t}
$$

On the other hand, the probability of calling spinning reserve may decrease the total operating cost. In order to find SRP in usually of a day scheduling it is needed to study in a larger horizon like a week or a month. In this paper $\mathrm{SRC}_{\mathrm{t}}$ as the total spinning reserve capacity is defined by Eq. (8).

$$
S R C_{t} \geq S R P_{t} \times S R R_{t}
$$

Where $\mathrm{SRP}_{\mathrm{t}}$ is the spinning reserve probability. Fig. 1 presents the forecasted probability of calling spinning reserve for the first day of year which has been used in this paper[10],[16].

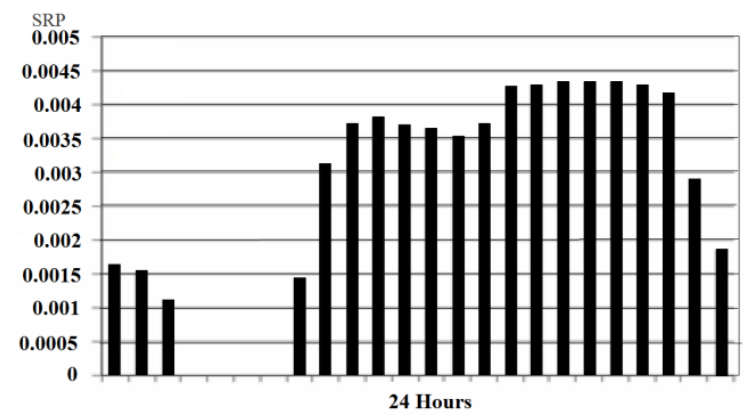

Figure 1. Forecasted probability that spinning reserve is called and generated

\section{B Primary frequency regulation constraints}

In addition to the above constraints in previous subsection, a unit commitment considering primary frequency regulation is subject to the following constraints:

- Primary reserve saturation level

In this paper, contingencies is defined by lose of one generating unit. This implies that following each contingency, only negative frequency deviations will occur. Fig. 2 illustrates the relation between primary reserve and frequency deviation for an arbitrary unit $i$. the upper primary reserve bound is the maximum output of unit $i$ under primary frequency regulation minus unit output, defined by either the unit frequency-regulation ramp limit, $r_{i}^{p r-\max }$, or by unit spinning capacity limit, $g_{i}^{\max }-g_{i t}$, whichever is smaller[3],[5],[6]. Unit frequency-regulation ramp limit is the maximum reserve that a unit can produce within 10 seconds following a contingency[6].

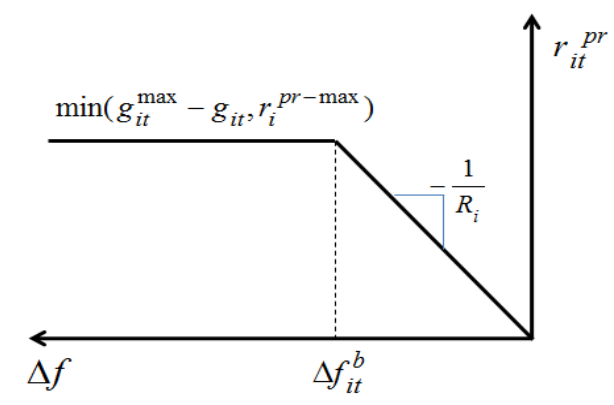

Figure 2. Primary reserve characteristic

In the other words, the relation between primary reserve and frequency deviation is linear according to governor droop of unit before the vertical line $\Delta f=\Delta f_{i}^{b}$, and for frequency deviation more than $\Delta f_{i}^{b}$, it is restricted to either unit spinning capacity limit or ramp up limit. This saturation level of primary reserve can be expressed by Eq. (9).

$$
v_{i t} \cdot v_{i t}^{p r} \leq \min \left(g_{i}^{\max }-g_{i t}, r_{i}^{p r-\max }\right)
$$

- Enough primary frequency control reserve

balancing of Generation and demand under each of the order of contingency is the major target of frequency reserves scheduling, so the remaining healthy generating units must provide enough reserve to make up for the lost generation under any contingency situation, occurring during any time interval $t$ of the scheduling horizon. These requirements for primary reserve can be satisfied by Eq. (10).

$$
\sum_{i=1, i \neq j}^{N} u_{i t} \cdot v_{i t} \cdot r_{i t}^{p r} \geq u_{j t} \cdot g_{j t}
$$

Obviously, the above inequation restricts the primary frequency regulation to a lower limit.

- maximum allowed frequency deviation

According to presented details in section 2, to avoid load shedding by under-frequency relays, the frequency deviations must be limited to allowed system frequency deviation as follows: 


$$
|\Delta f| \leq\left|\Delta f_{c r}\right|
$$

Eq. (11) can be satisfied considering a linear constraint as Eq. (12)[17].

$$
u_{i t} \cdot v_{i t} \cdot r_{i t}^{p r} \leq \frac{\Delta f_{c r}}{R_{i}}
$$

- Maximum generation deviation level

Finally, the scheduled primary regulation reserve for unit $i$, must be greater than or equal to the maximum generation deviation relative to the pre-contingency level over all contingencies[16].

$$
u_{i t} \cdot v_{i t} \cdot \Delta g_{i t}^{j} \leq r_{i t}^{p r}, j \neq i
$$

This important was not considered in[5] and[14] while the final solution of scheduling problem will not be practical if this inequation is not satisfied. It should be noted that this is a nonlinear constraint.

\section{Objective function}

The achievement of simultaneous unit commitment with primary frequency regulation requirements in power system operation consists of two terms. Including both operating costs and costs of supplying primary reserve services. Which depend on demand and are subject to the aforementioned constraints, i.e. how to allocate the required load demand among units. These requirements can be expressed in an objective function subject to the constraints considered. The objective function that seeks to minimize the total cost is given by Eq. (14). Here operating costs include fuel cost of generating units, the startup cost of committed units and the shutdown cost of decommitted units.

$$
\begin{aligned}
& \operatorname{Min}: \sum_{t=1}^{H} \sum_{i=1}^{N} C^{F}\left(g_{i t}\right) \cdot u_{i t}+\sum_{t=1}^{H} \sum_{i=1}^{N} S U C_{i t} \cdot u_{i t} \cdot\left(1-u_{i, t-1}\right) \\
& +\sum_{t=1}^{H} \sum_{i=1}^{N} S D C_{i t} \cdot u_{i, t-1} \cdot\left(1-u_{i t}\right)+\sum_{t=1}^{H} \sum_{i=1}^{N} C^{p r}\left(r_{i t}^{p r}\right) \cdot u_{i t} \cdot v_{i t}
\end{aligned}
$$

The energy and reserve cost functions are typically expressed, respectively, quadratic polynomial and linear as defined in (15) and (16)[3],[16].

$$
\begin{gathered}
C^{F}\left(g_{i t}\right)=c_{i}+b_{i} \cdot g_{i t}+a_{i} \cdot g_{i t}^{2} \\
C^{p r}\left(r_{i t}^{p r}\right)=q_{i t} \cdot r_{i t}^{p r}
\end{gathered}
$$

Although minimum up time, minimum down time, ramp up and ramp down rate limit and shut down cost of units can be considered in optimization process, However to simplicity just start-up cost constraint has been considered in this paper.

\section{Optimization Method}

The type of simultaneous scheduling of energy and primary reserve is a Mixed Integer Non Linear Programming (MINLP) problem[16]. Where the binary variables are the representation of on/off status and continual variables represent the values of generation and reserves. In solving this problem an important challenges arise: the fact that value of primary reserve of unit $i$ must lie on the piece-wise curve imposed by Fig. 1, which is described by four decision criterions including the spinning reserve capacity of unit $i$, ramp up limit of unit $i$, the allowed frequency deviation and the corresponding frequency deviation is another. GAMS (Generalized Algebra Modeling System) is one of most popular commercial software in algebraic modeling of optimization problems including integer and continual variables. It is a computer environment that can easily be used to model and solve optimization problems. GAMS is specially design for linear, nonlinear and mixed integer optimization and has been commonly applied to solve power system optimization problems[19],[20]. Even though it is required some effort to become familiar with GAMS, in following we will see that this is a very comprehensive and powerful tool to solve unit commitment with primary frequency regulation constraints.

\section{Case Study and Simulation Results}

In this paper, it is assumed that each unit can lie in three modes which are: 1) unit is shut down, 2) unit is turn on and provides energy but does not participate in frequency control, 3) unit is turn on and participates in both generation and primary frequency regulation as shown in Table 1. Notice, a shut down unit cannot participate in primary frequency control.

Table1. Status of Binary Variables

\begin{tabular}{ccc}
\hline $\mathrm{u}$ & $\mathrm{v}$ & feasibility \\
\hline 0 & 0 & $\sqrt{ }$ \\
\hline 1 & 0 & $\sqrt{ }$ \\
\hline 0 & 1 & $\times$ \\
\hline 1 & 1 & $\sqrt{ }$ \\
\hline
\end{tabular}

Table 2. Demand Characteristic versus Time of 17-Unit System

\begin{tabular}{ccccc}
\hline Hour & 1 & 2 & 3 & 4 \\
\hline Load(MW) & 1531 & 1439 & 1371 & 1348 \\
\hline Hour & 5 & 6 & 7 & 8 \\
\hline Load(MW) & 1348 & 1371 & 1691 & 1965 \\
\hline Hour & 9 & 10 & 11 & 12 \\
\hline Load(MW) & 2170 & 2193 & 2193 & 2170 \\
\hline Hour & 13 & 14 & 15 & 16 \\
\hline Load(MW) & 2170 & 2170 & 2125 & 2148 \\
\hline Hour & 17 & 18 & 19 & 20 \\
\hline Load(MW) & 2262 & 2285 & 2285 & 2193 \\
\hline Hour & 21 & 22 & 23 & 24 \\
\hline Load(MW) & 2079 & 1896 & 1668 & 1439 \\
\hline
\end{tabular}

In next step the proposed method to solve simultaneous scheduling of energy and primary reserve considering spinning reserve uncertainty is tested on an isolated power 
system without frequency regulation support from neighboring systems including 17 -generating units. This system is scheduled over a 24 -h horizon. The 24 -h load characteristics are those corresponding to the first day of the year from the IEEE reliability test system shown in Table $2[21]$.

Generator data are taken from[6] shown in Table 3. All units have a regulation droop of $5 \%$ with a system nominal frequency of $50 \mathrm{~Hz}$ and a maximum allowed frequency deviation of $500 \mathrm{mHz}$.

It is also assumed that price of primary reserve are set to one tenth of the linear generation cost components, $b_{i}$.

Table 3. Test System Data

\begin{tabular}{ccccccccc}
\hline & $a_{i}$ & $b_{i}$ & $c_{i}$ & $g^{\min }$ & $g^{\max }$ & $r^{p r-m a x}$ & $q_{i}$ & $S U C_{i}$ \\
\hline Unit & $£ / M W h^{2}$ & $£ / M W h$ & $£ / h$ & $M W$ & $M W$ & $M W$ & $M W$ & $£$ \\
\hline $\mathbf{A}$ & 0.0009 & 9.8 & 200 & 100 & 330 & 26 & 0.98 & 5552 \\
\hline B & 0.0009 & 10.7 & 157 & 130 & 298 & 25 & 1.07 & 4987 \\
\hline $\mathbf{C}$ & 0.0009 & 13.6 & 800 & 120 & 154 & 20 & 1.36 & 2453 \\
\hline $\mathbf{D}$ & 0.00146 & 14.8 & 547 & 95 & 123 & 15 & 1.48 & 989 \\
\hline E & 0.00132 & 15.2 & 532 & 37 & 234 & 16 & 1.52 & 2675 \\
\hline F & 0.00504 & 16.1 & 532 & 37 & 246 & 15 & 1.61 & 2985 \\
\hline G & 0.00504 & 16.1 & 590 & 17 & 91 & 5 & 1.61 & 3334 \\
\hline H & 0.0324 & 16.4 & 612 & 25 & 95 & 5 & 1.64 & 3789 \\
\hline I & 0.0324 & 17.1 & 580 & 54 & 274 & 21 & 1.71 & 2976 \\
\hline J & 0.0047 & 17.1 & 377 & 58 & 276 & 19 & 1.71 & 2543 \\
\hline K & 0.0035 & 17.7 & 670 & 14 & 82 & 5 & 1.77 & 3245 \\
\hline L & 0.02118 & 18.3 & 910 & 22 & 159 & 20 & 1.83 & 2650 \\
\hline M & 0.00558 & 19.5 & 155 & 55 & 114 & 10 & 1.95 & 500 \\
\hline N & 0.00088 & 20 & 170 & 64 & 126 & 15 & 2 & 500 \\
\hline O & 0.00094 & 22.1 & 658 & 14 & 100 & 10 & 2.21 & 2870 \\
\hline P & 0.00244 & 24.8 & 297 & 52 & 118 & 10 & 2.48 & 701 \\
\hline Q & 0.00076 & 25.2 & 103 & 28 & 62 & 5 & 2.52 & 632 \\
\hline & & & & & & & \\
\hline
\end{tabular}

There are several MINLP solvers in GAMS software[22]. Surveys indicate that some of them such as BONMIN, OQNLP and KNITRO are capable to solve simultaneous unit commitment with primary frequency regulation constraints.

We choice BONMIN solver to solve optimization problem due to its low elapsed time and in addition it was most powerful from cost minimization point of view.

Table 4 illustrates the generation levels and primary reserves for 17-unit, multi-period obtained using the proposed optimization method. Note, that we only show six representative time periods, namely, 1, 7, 13, 19, 23 and 24 of the full 24-h schedule.

As a clear result, the values of Table 4 indicate that simultaneous scheduling need more units to be turned on versus increasing demand to satisfy the load and enough primary reserve.

In addition, Simulation results also indicate that depending on the problem parameters settled frequency deviation can fall before or after the break frequency deviation of the frequency regulation curve of a unit. This means that in general, it is not predictable whether the binary variables $u_{i t}$ and $v_{i t}$ will take a value of 0 or 1 . This matter is demonstrated looking at columns of 23th hour in Table 3. In this hour units C, D \& L produce 18, 14 and $18 \mathrm{MW}$ respectively for primary reserves whiles their primary saturation levels are 20,15 and $20 \mathrm{MW}$ respectively.

Table 4. Units Output Power and Primary Reserve Level

\begin{tabular}{cccccccccccccc}
\hline H & \multicolumn{1}{c}{$\mathbf{1}$} & \multicolumn{1}{c}{$\mathbf{7}$} & \multicolumn{1}{c}{$\mathbf{1 3}$} & \multicolumn{1}{c}{$\mathbf{1 9}$} & \multicolumn{2}{c}{$\mathbf{2 3}$} & \multicolumn{2}{c}{$\mathbf{2 4}$} \\
\hline Unit & $g_{i}$ & $r^{p r}$ & $g_{i}$ & $r^{p r}$ & $g_{i}$ & $r^{p r}$ & $g_{i}$ & $r^{p r}$ & $g_{i}$ & $r^{p r}$ & $g_{i}$ & $r^{p r}$ \\
\hline $\mathbf{A}$ & 161 & 26 & 181 & 26 & 216 & 26 & 216 & 26 & 181 & 26 & 161 & 26 \\
\hline $\mathbf{B}$ & 162 & 0 & 182 & 25 & 217 & 25 & 217 & 25 & 182 & 25 & 162 & 25 \\
\hline $\mathbf{C}$ & 134 & 20 & 0 & 0 & 134 & 20 & 134 & 20 & 134 & 18 & 134 & 20 \\
\hline $\mathbf{D}$ & 108 & 15 & 0 & 0 & 108 & 15 & 108 & 15 & 108 & 14 & 108 & 15 \\
\hline $\mathbf{E}$ & 171 & 16 & 0 & 0 & 218 & 16 & 218 & 16 & 191 & 16 & 171 & 16 \\
\hline $\mathbf{F}$ & 172 & 15 & 0 & 0 & 227 & 15 & 227 & 15 & 192 & 15 & 172 & 15 \\
\hline $\mathbf{G}$ & 86 & 5 & 0 & 0 & 86 & 5 & 86 & 5 & 86 & 5 & 86 & 5 \\
\hline $\mathbf{H}$ & 60 & 0 & 60 & 0 & 0 & 0 & 90 & 5 & 60 & 0 & 60 & 0 \\
\hline $\mathbf{I}$ & 154 & 21 & 96 & 21 & 86 & 21 & 126 & 21 & 87 & 21 & 62 & 21 \\
\hline $\mathbf{J}$ & 168 & 19 & 188 & 19 & 223 & 19 & 223 & 19 & 188 & 19 & 168 & 19 \\
\hline $\mathbf{K}$ & 0 & 0 & 0 & 0 & 0 & 0 & 77 & 5 & 48 & 0 & 48 & 0 \\
\hline $\mathbf{L}$ & 0 & 0 & 0 & 0 & 0 & 0 & 139 & 20 & 104 & 18 & 91 & 0 \\
\hline $\mathbf{M}$ & 0 & 0 & 0 & 0 & 104 & 10 & 104 & 10 & 104 & 10 & 104 & 10 \\
\hline $\mathbf{N}$ & 0 & 0 & 0 & 0 & 111 & 15 & 111 & 15 & 111 & 15 & 111 & 15 \\
\hline $\mathbf{O}$ & 0 & 0 & 0 & 0 & 0 & 0 & 90 & 10 & 57 & 0 & 57 & 0 \\
\hline $\mathbf{P}$ & 0 & 0 & 0 & 0 & 0 & 0 & 91 & 10 & 85 & 0 & 85 & 0 \\
\hline $\mathbf{Q}$ & 0 & 0 & 0 & 0 & 0 & 0 & 28 & 5 & 45 & 5 & 45 & 0 \\
\hline & & & & & & & & & & & \\
\hline
\end{tabular}

It is important to reemphasize that Table 3 shows some generating units such as $\mathrm{B} \& \mathrm{H}$, (in Hour=1), are participating in generation but they don't provide primary frequency control reserve whiles in[3] all of participating units must common to provide primary reserve. This is due to considering a new binary variable, $v_{i}$, that defines on or off status of governor of a generating unit. This important improve problem formulation of simultaneous scheduling.

Total operating cost including both generation and primary reserve over the 24 hour period is equal to 842997.5£.

\section{Conclusions}

In this paper a simultaneous multi period unit commitment problem subject to primary regulation reserve constraints is formulated. In the other hand, the spinning reserve probability as a practical constraint has been taken into account. This problem has not received as much attention in the previous works. In addition, for committed generating units three alternatives are defined including participating in both generation and primary frequency control, just participating in generation and finally disaffiliation in 
generation and supplying primary reserve. It is also not considered in the previous researches. In this paper, only credible contingencies, negative frequency deviation following each contingency have been considered.

Finally, the proposed optimization method has been implemented on a test system including 17 generating units, a well-known test system. Results indicate that the final solution is applicable.

Although the methodology presented has been examined on a small isolated power utility but it is practical even for large systems.

\section{ACKNOWLEDGEMENTS}

We are thankful to South Kerman Electrical Distribution Company for their support and also many thanks go in particular to Dr.Mashhadi, Dr.Javidi and Dr.Ghazizadeh for their valuable work reported in[5].

\section{Numencalture}

A. Continuous Variables

$g_{i t}$ Scheduled generation of unit $i$ in the pre contingency state during time period $t$

$r_{i t}^{p r}$ Scheduled primary reserve of unit $i$ during time period $t$

$\Delta f_{i}^{b} \quad$ Break Frequency deviation of unit $i$

$\Delta g_{i}^{j} \quad$ Generation deviation in output of unit $i$ after contingency due to outage of unit $j$

B. 0/1 Binary Variables

$u_{i t}$ A variable defining the operation status of generator $\mathrm{i}$ during time period $t$ (equals 1 if the unit is on and zero if it is off)

$v_{i t}$ A variable defining the operation status of governor of generating unit $i$ (equals 1 if the governor be in active mode and zero if not)

$y_{i t}$ Equals 1 if unit $i$ turns on during time period and equals zero if it does not.

$z_{i t}$ Equals 1 if unit $i$ turns off during time period $\mathrm{t}$ and equals zero if it does not

C. Parameters

$R_{i}$ Governor droop of unit $i$

$a_{i}$ Unit $i$ quadratic generation cost parameter

$b_{i}$ Unit $i$ linear generation cost parameter

$c_{i}$ Unit $i$ fixed generation cost

$C^{F}\left(g_{i t}\right)$ Fuel cost

$C^{p r}\left(r_{i t}^{p r}\right)$ Cost of supplying primary reserve

$d_{t}$ System demand during time period $t$

$g_{i}^{\min }$ Minimum possible generation output of unit $i$

$g_{i}^{\max }$ Maximum possible generation output of unit $i$

$H$ Unit commitment horizon
$M D_{i}^{\text {OFF }}$ Duration during which the ith unit is continuously on

$M D_{i}^{O N}$ Duration during which the ith unit is continuously off

$N$ Number of units

$q_{i t}^{p r}$ Primary reserve price during time period $t$ for unit $i$

$q_{i t}^{s e}$ Secondary reserve price during time period $t$ for unit $i$

$q_{i t}^{t r} \quad$ Tertiary reserve price during time period $t$ for unit $i$

$R U R_{i}$ Ramp up rate limit of unit $i$

$R D R_{i}$ Ramp down rate limit of unit $i$

$r^{p r-\max }$ Unit $i$ ramp-up limit under primary regulation

$S D C_{i t}$ Shut down cost of unit $i$ at hour $t$

$S R C$ Spinning reserve capacity

$S R R$ Spinning reserve requirement

$S U C_{i t}$ Start up cost of unit $i$ at hour $t$

$T_{i}^{D}$ Minimum down time limit of unit $i$

$T_{i}^{O N}$ Minimum up time limit of unit $i$

$\Delta f_{c r}$ Critical frequency deviation allowed

\section{REFERENCES}

[1] A.J.Wood, B.F.Wollenberg, "Power generation operation and Control " (2nd Ed. New York: Wiley, 1996).

[2] R. J. Koessler, J. W. Feltes, J. R. Willis, “A methodology for management of spinning reserves requirements", Proc. IEEE Power Eng. Soc. Winter Meeting, vol. 1, Feb. 1999, pp. 584-589.

[3] Jose' F.Restrepo, Francisco D.Galiana, "Unit commitment with primary frequency regulation constraints" ,IEEE Trans.Power Syst. ,vol.20, no.4,pp.1836-1843, Nov 2005.

[4] N. Jalleli, D. N. Ewart, L. H. Fink, A. G. Hoffmann, "Understanding automatic generation control", IEEE Trans. Power Syst., vol. 7,no. 3, pp. 1106-1112, Aug. 1992.

[5] M.R.Mashhadi, M.H.Javidi, M.S.Ghazi zadeh, "The impacts of capabilities and constraints of generating units on simultaneous scheduling of energy and primary reserve", Electrical Engineering, pp. 1-10. doi:10.1007/s00202-010, 2011

[6] J.W.O'Sullivan, M.J.O. Malley, "A new methodology for the provision of reserve in an isolated power system", IEEE Trans.Power Syst., vol.14, no.2, pp.519-523, May 1999.

[7] K.A.Papadogiannis, N.D.Hatziagyriou, "Optimal allocation of primary reserve services in energy markets", IEEE Trans.Power Syst. vol.19, no.1, pp.519-523, Feb.2004.

[8] Fransisco D.Galiana, Franccois Bouffard, Jose M.Arroyo, Jose' F.Restrepo, "Scheduling and pricing of coupled energy and primary, secondary, and tertiary reserves", Proceedings of the IEEE, pp.1970-1984, vol.93, no.11, 2005.

[9] M.Pourakbar-Kasmaie, M.RashidiNejad, "A novel unit commitment technique considering prohibited zones", Journal of applied science, vol.9.n.16. pp 2962-2968, 2009. 
[10] A.Abdollahi, M.Ehsan, M.Rashidi-Nejad, M.Pourakbar-Kasmaie, "cost-based unit commitment considering prohibited zones and reserve uncertainty", Journal of IREE, vol.4, N.3, pg:425-433,May-June 2009.

[11] S.J.Huang, C.L.Huang. "Application of Genetic-Based Neural Network to Thermal Unit Commitment", IEEE Trans Power Syst.12 (1997) 654-660.

[12] C.Wang, SM.Shahidehpour. "Affect of Ramp-Rate Limits on Unit Commitment and Economic Dispatch". IEEE Trans on Power Syst.8 (3) (1993) 1341-1350.

[13] Vo Ngoc Dieu, Weerakorn Ongsakul. "Ramp Rate Constrained Unit Commitment by Improved Priority List and Augment Lagrange Hopfield Network, EPSR.78(3) (2008) 291-301.

[14] Bruce A. Grey, Ghadir Radman, Arun Sekar, "Determination of Spinning Reserve Deployment using an Extended Economic Dispatch to Include Line Flow Limits and Primary Frequency Regulation". 39th Southeastern Symposium on System Theory, Mercer University, March 4-6, 2007.

[15] Fushan Wen, A.K.David, "Coordination of bidding strategies in day-ahead energy and spinning reserve markets", Jour nal of Electrical power and energy systems, vol.24, pg:251-261, April.2002.

[16] H.Y. Yamin, Q.El-Dwairi, S.M.Shahidehpour,“ A new approach for GenCos profit based unit commitment in day-ahead competitive electricity markets considering reserve uncertainty”. EPES.29(2007) 609-616.

[17] M.Rouholamini, M.Rashidi-nejad, S.Esmaeili, "A new methodology to solve joint energy and primary reserve scheduling" 5 th power control and optimization conference. UAE, Doubai., in press.

[18] Jose Restrepo, "Unit Commitment with Primary Frequency Regulation Constraint in Electric Power System”, Ph.D. dissertation, department of electrical and computer of McGill University, February 25, 2005.

[19] D. Chattopadhyay, "Application of general algebraic modeling system to power system optimization", IEEE Transactions on Power Systems, vol. 14, no. 1, February 1999, pp. $15-22$.

[20] Bhujanga B Chakrabarti, Doug Goo win, Nirmal-Kumar C Nair, "Power system congestion - in search of an index from LP basis matrices", Power Engineering Society General Meeting, IEEE, june 2007.

[21] Reliability test system task force, The IEEE reliability test system- 1996, IEEE Trans. Power Syst., vol. 14, no. 3, pp. 1010 1020, Aug. 1999.

[22] GAMS solver manuals, http://www.gams .com/solvers. 